\title{
UNSMOOTHABLE DIFFEOMORPHISMS ON HIGHER DIMENSIONAL MANIFOLDS
}

\author{
JENNY HARRISON
}

\begin{abstract}
Examples of $C^{r}$ diffeomorphisms not topologically conjugate to $C^{s}$ diffeomorphisms $(s>r)$ are constructed on manifolds of dimension $\neq 1,4$.
\end{abstract}

A $C^{r}$ diffeomorphism is said to be "unsmoothable" if it is not topologically conjugate to any $C^{s}$ diffeomorphism for some $s>r$. In [2] examples of unsmoothable diffeomorphisms of surfaces are described for integers $r$ and $s$. The present paper extends the construction to higher dimensional manifolds with the exception of dimension four and to all positive real numbers $r$ and $s$. In fact, for certain homeomorphisms there is a topological invariant in $[0, \infty]$ defined for every $r>0$. The invariant being finite is a necessary and sufficient condition for the homeomorphism to be topologically conjugate to a $C^{r}$ diffeomorphism.

Fundamental to the construction of these examples is the Packing Lemma: Any infinite collection of geometric $n$-disks with finite total volume may be arranged disjointly in $\mathbf{R}^{n}$ to converge to one point. (This is equivalent to a statement about infinite series. If $\Sigma a_{k}$ has the property that $\Sigma a_{k}^{n}<\infty$ there exists a convergent subseries $\Sigma a_{k_{i}}<\infty$ such that the sum of the terms in between, $\sum_{j=k_{i}}^{k_{i}} a_{j}$, tends to zero as $i$ gets large.)

The unsmoothable diffeomorphisms defined on a manifold $M^{n+1}$ form a dense subset of the topological group of $C^{r}$ diffeomorphisms of $M^{n+1}$ with the $C^{0}$ topology. As in [2] unsmoothable diffeomorphisms may be suspended to form an unsmoothable flow or foliation on $M^{n+1} \times S^{1}$.

I would like to thank M. Freedman for his helpful suggestions and especially $\mathrm{L}$. Marcus for telling me about this problem to begin with.

1. Higher dimensional examples. Let $n$ be an integer $>1$ and $\neq 3$, and let $M^{n+1}$ be a $C^{\infty}$ manifold. Define $D^{n}$ to be the $n$-fold product of the closed unit interval $[0,1]$, and $S^{1}$ to be the real numbers modulo 1 . Let $\psi$ be a $C^{\infty}$ embedding of $D^{n} \times S^{1}$ in $\mathbf{R}^{n+1}$ which links each $x \times S^{1}$ once around the $(n+1)$ st axis of $\mathbf{R}^{n+1}$. We will define an unsmoothable $C^{r}$ diffeomorphism $f$

Received by the editors June 13, 1977.

AMS (MOS) subject classifications (1970). Primary 57D50, 58C99; Secondary 57D30, 58F99. 
of $\mathbf{R}^{n+1}$ which is the identity outside the image of $D^{n} \times S^{1}$. Then $f$ can be glued to a local chart of $M^{n+1}$ and extended by the identity to the rest of $M^{n+1}$ to form an unsmoothable $C^{r}$ diffeomorphism of $M^{n+1}$. (See Corollary 1.)

Let $P_{k}$ be a sequence of points in $D^{n}$ converging to $P$ in $D^{n}$. Denote the circle $\psi\left(P_{k} \times S^{1}\right)$ in $\psi\left(D^{n} \times S^{1}\right)$ by $S_{k}$. Suppose we are given rotations $f_{k}$ of each of these circles $S_{k}$ with rotation number $\rho_{k}$. We are interested in extending the $f_{k}$ to a diffeomorphism of $\mathbf{R}^{n+1}$ which is the identity outside disjoint tubular neighborhoods $N_{k}$ of the $S_{k}$. If $\rho_{k} \rightarrow 0$ there are always such extensions which are continuous. We would like to choose one so that some member of its topological conjugacy class is "as smooth as possible". This highest possible degree of smoothness is, in fact, dictated by the original rotation numbers $\rho_{k}$.

THEOREM 1. Suppose $r>0$. There exist homeomorphisms $f$ and $h$ of $\mathbf{R}^{n+1}$ such that $f \mid S_{k}=f_{k}$, $f$ is the identity outside $\cup N_{k}$ and $h^{-1}{ }^{-1}$ is $C^{r}$ if and only if $\Sigma\left|\rho_{k}\right|^{n / r}<\infty$.

Thus strictly topological information tells us how smooth the extension can be made.

The following lemmas will be useful for the proof of this theorem.

LEMMA 1. If $\Sigma a_{k}$ is a convergent series of positive terms then there exists an unbounded positive sequence of numbers $N_{k}$ such that $\Sigma N_{k} a_{k}<\infty$.

Proof. Let $N_{k}=\min \left\{i: \sum_{n=k}^{\infty} a_{n}>\sum_{n=i}^{\infty} 1 / 2^{n}\right\}$. The result follows since

$$
\sum N_{k} a_{k}<\sum k / 2^{k}<\infty \text {. }
$$

It is an interesting exercise to try and arrange disks of radius $1 / n$ in $\mathbf{R}^{2}$ to converge to one point. They cannot be lined up, but somehow it is possible to arrange them in stacks of more or less equal height which can then be broken into stacks of diminishing height using Lemma 1.

Lemma 2 (PACKIng Lemma). Suppose $\sum a_{k}^{n}<\infty$ and $n \geqslant 1$. Then there exist disjoint $n$-cubes with edges of length $a_{k}$ in $\mathbf{R}^{n}$ whose centers converge to one point.

Proof. The lemma is trivial for $n=1$ and proceeds by induction for $n>1$. We can assume that the sequence $a_{k}^{n}$ and hence $a_{k}$ decreases monotonically.

The $n$-cubes $A_{k}$ are first placed in an $n$-box parametrized by

$$
\left[0, a_{1}\right]^{n-2} \times[0, \infty) \times\left[0,2 a_{1}\right] .
$$

Let $a_{t_{i}}$ be the subsequence of the $a_{k}$ where $t_{1}=1$ and

$$
t_{i}=\max \left\{t \in Z: \sum_{j=t_{i-1}}^{t-1} a_{j}<2 a_{1}\right\} .
$$


Suppose $t_{i}<k<t_{i+1}$. Identify $A_{k}$ with the $n$-cube

$$
\left[0, a_{k}\right]^{n-2} \times\left[\sum_{j=1}^{i-1} a_{t_{j}}, \sum_{j=1}^{i-1} a_{t_{j}}+a_{k}\right] \times\left[\sum_{j=t_{i}}^{k-1} a_{j}, \sum_{j=t_{i}}^{k} a_{j}\right]
$$

This divides the sequence of $n$-cubes $A_{k}$ into stacks with the bottom $n$-cube in the $i$ th stack being $A_{t_{i}}$. Each stack has "height" in the last factor between $a_{1}$ and $2 a_{1}$.

We show that the $n$-box into which the $n$-cubes are now packed is finite, in some sense. Let $\beta=\sum_{i=1}^{\infty} a_{t_{i}}^{n-1}$, the sum of the "areas" of the $(n-1)$-faces of the cubes at the bottom of each stack, and $\tau=\sum_{i=2}^{\infty}\left(a_{t_{i}-1}\right)^{n-1}$, the sum of the areas of the $(n-1)$-faces at the top of each stack. Then, clearly, $\tau \cdot a_{1}<\sum a_{k}^{n}$ $<\infty$. Hence $\tau<\infty$. But, since $a_{k}$ decreases monotonically, $\beta<\tau+a_{1}^{n-1}$. Hence $\beta<\infty$.

We now apply Lemma 1 to break each $i$ th stack into $N_{i}$ new stacks of diminishing height so that the total area at the bottom of the new stacks is still finite. The number of $n$-cubes in the original $i$ th stack is $t_{i+1}-t_{i}$ and $\left(t_{i+1}-t_{i}\right) / N_{i}$ is an integer $Z_{i}$ with remainder term $r_{i}$. Make $N_{i}$ shorter stacks out of the $i$ th stack by putting $Z_{i}+1$ of the $n$-cubes into each of $r_{i}$ new stacks and $Z_{i} n$-cubes into the others.

Finally, apply the induction hypothesis to disjointly arrange the $(n-1)$ faces at the bottom of each stack and hence the stacks themselves to converge to one point.

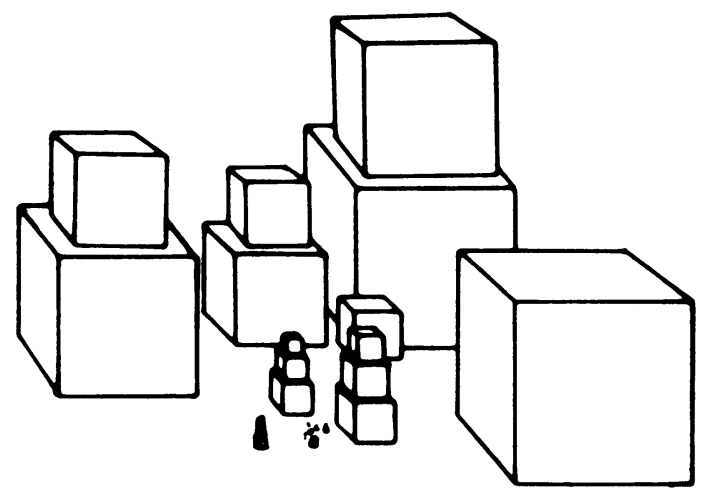

Later we will use this lemma to sufficiently separate the circles $S_{k}$ so then an extension $f$ of the $f_{k}$ can be made $C^{r}$-smooth by a topological conjugacy.

LEMMA 3. Any homeomorphism $h: \mathbf{R}^{n+1} \rightarrow \mathbf{R}^{n+1}, n \neq 3$, can be approximated by a $C^{\infty}$ diffeomorphism.

Proof. According to E. H. Connel [1], $h$ can be approximated by a $C^{\infty}$ diffeomorphism if and only if $h$ is stable. But Kirby's annulus theorem [3] is 
equivalent to the fact that all (orientation preserving) homeomorphisms are stable, $n \neq 3$.

Proof OF TheOReM 1. Suppose homeomorphisms $f$ and $h$ of $\mathbf{R}^{n+1}$ exist such that $f^{\prime}=h f h^{-1}$ is $C^{r}$ and $f$ extends the given rotations in the prescribed manner. Choose a disk $D_{0}^{n} \subset \overline{\mathbf{R}^{n}}$ where $\overline{\mathbf{R}^{n}}=\left\{\left(x_{1}, \ldots, x_{n}\right) \in \mathbf{R}^{n}: x_{n}>0\right\}$ is the upper half of $\mathbf{R}^{n}$. Let $\gamma$ be a $C^{\infty}$ diffeomorphism,

$$
\gamma: \overline{\mathbf{R}^{n}} \times S^{1} \bigcup_{\mathbf{R}^{n-1} \times S^{1}} \mathbf{R}^{n-1} \times D^{2} \rightarrow \mathbf{R}^{n+1}
$$

identifying $\gamma\left(D_{0}^{n} \times S^{1}\right)$ with $\psi\left(D^{n} \times S^{1}\right)$. By Lemma 3 we can assume that $h$ is close to the identity. Hence each circle $h S_{k}$ still winds once around the central core $\gamma\left(\mathbf{R}^{n-1} \times D^{2}\right)$.

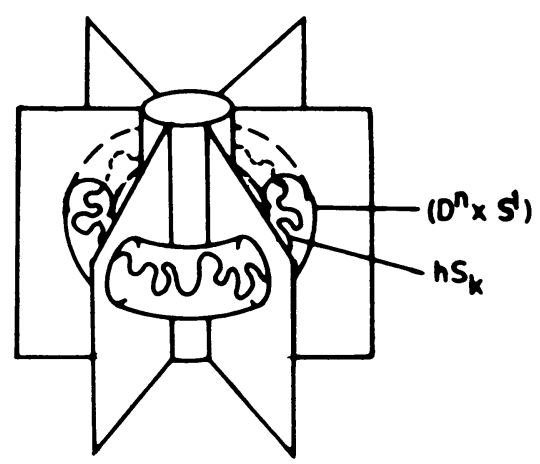

Let $\pi_{2}: \overline{\mathbf{R}^{n}} \times S^{1}$ be the projection onto the second factor and define $\pi$ to be the composition $\pi_{2} \circ \gamma^{-1}$.

Now proceed as in [2], replacing the projection map $\pi_{2}$ by the map $\pi$ just defined. We give here a brief sketch.

(1) Define functions $l_{k}: S^{1} \rightarrow h S_{k}$ by $l_{k}(x)=\sup \left\{h S_{k} \cap \gamma\left(\overline{\mathbf{R}^{n}} \times x\right)\right\}$ and $m_{k}: h S_{k} \rightarrow S_{k}$ by $m_{k}(x)=\inf \left\{y \in L_{k}: y \geqslant x\right\}$ where $L_{k}$ is the image of $l_{k}$.

(2) The composition $f^{\prime \prime}=\pi m_{k} f^{\prime} l_{k}$ satisfies the following properties:

(i) $\left|f^{\prime \prime} \pi(x)-\pi(x)\right|<\left|\pi f^{\prime}(x)-\pi(x)\right|$ for all $x$ in $L_{k}$.

(ii) There is a finite union $A_{k}$ of disjoint closed intervals in $S^{1}$ of total length $>1 / 6$ such that $\left|f^{\prime \prime}(a)-a\right| \geqslant \rho_{k} / 4$ for all $a \in A_{k}$.

(3) A line segment $\mu_{k}$ is found with endpoints on the boundary of $h N_{k}$ and interior contained in $\gamma\left(\overline{\mathbf{R}^{n}} \times x\right) \cap h N_{k}$. Furthermore, $\mu_{k}$ has minimal length (considered over all such line segments with $x$ in $A_{k}$ ) and contains a point which is moved around the core circle at least $\rho_{k} / 4$.

(4) By Fubini's theorem, the volume of $h N_{k} \geqslant$ constant $\left|\mu_{k}\right|^{n}$. Hence $\sum\left|\mu_{k}\right|^{n}<\infty$.

(5) By a repeated application of the mean value theorem, there exists a point $x_{k}$ in $\mu_{k}$ such that $\left|\partial^{s}\left(\pi f^{\prime}-\pi\right)\left(x_{k}\right) / \partial x^{s}\right|>\left|\rho_{k}\right| / 4\left|\mu_{k}\right|^{s}$ for all integers $s<r$. Since all the partial derivatives tend to zero as $k$ gets large, $\left|\rho_{k}\right| / 4\left|\mu_{k}\right|^{s}$ $\rightarrow 0$. Therefore, from step $4, \sum_{k=1}^{\infty}\left|\rho_{k}^{n / s}\right|<\infty$. 
Now suppose $r=s+\alpha$ where $0<\alpha<1$ and $s$ is an integer. Since $f^{\prime}$ is $C^{r}$, the Hölder inequality holds for the sth partial derivatives and constant $\alpha$.

Thus there are points $x_{k}$ in $\mu_{k}$ and $y_{k}$ in the boundary of $\mu_{k}$ such that

$$
\begin{aligned}
& \left|\rho_{k}\right| / 4\left|\mu_{k}\right|^{s}<\left|\partial^{s}\left(\pi f^{\prime}-\pi\right)\left(x_{k}\right) / \partial x^{s}\right| \\
& \quad=\left|\partial^{s}\left(\pi f^{\prime}-\pi\right)\left(x_{k}\right) / \partial x^{s}-\partial^{s}\left(\pi f^{\prime}-\pi\right)\left(y_{k}\right) / \partial x^{s}\right|<\lambda\left|x_{k}-y_{k}\right|^{\alpha}<\lambda\left|\mu_{k}\right|^{\alpha} \text {. } \\
& \text { Hence }\left|\rho_{k}\right|<\lambda\left|\mu_{k}\right|^{s+\alpha} \text { and }\left|\rho_{k}\right|^{n / s+\alpha}<\lambda\left|\mu_{k}\right|^{n} \text {. Therefore } \sum_{k=1}^{\infty}\left|\rho_{k}\right|^{n / r}<\infty \text {. }
\end{aligned}
$$

Conversely suppose $\Sigma\left|\rho_{k}^{n / r}\right|<\infty$. It follows from Lemma 1 that there exists a convergent positive series $a_{k}^{n}$ such that $\left|\rho_{k}^{n / r}\right| / a_{k}^{n} \rightarrow 0$. That is, $\left|\rho_{k}\right| / a_{k}^{r} \rightarrow 0$. According to the Packing Lemma there is a homeomorphism $H$ of the disk $D^{n}$ sending the chosen sequence of points $P_{k}$ to another convergent sequence of points $q_{k}$ which are the centers of disjoint $n$-disks $B_{k}$ with radius $a_{k}$, and $H$ is fixed on a neighborhood of the boundary of $D^{n}$. Consider the product of $H$ with the identity. This is a homeomorphism of the product $D^{n} \times S^{1}$ which only changes the relative positions of the circles $P_{k} \times S^{1}$, leaving them with the same geometric rotations as before. Now extend these rotations on $q_{k} \times S^{1}$ to a diffeomorphism of $B_{k} \times S^{1}$ by using a smooth bump function $\Phi$ of the unit $n$-disk which is zero on a neighborhood of the boundary of the disk and is 1 at the center. For instance, let

$$
g(x, t)=\left(x, t+\rho_{k} \Phi\left(x-q_{k}\right) / a_{k}\right)
$$

for $(x, t) \in B_{k} \times S^{1}$.

Finally extend $g$ to the identity outside of $B_{k} \times S^{1}$. Calculating the $s$ th partial derivatives of $g$ restricted to $B_{k} \times S^{1}$, it is easily verified (as in [2]) that this extension $g$ is a $C^{\infty}$ diffeomorphism away from the limit circle $q \times S^{1}$ and is $C^{s}$ at $q \times S^{1}$ since $\rho_{k} / a_{k}^{s} \rightarrow 0$ for $s \leqslant r$.

This completes the proof if $r$ is an integer.

Again suppose that $r=s+\alpha$ where $0<\alpha<1$ and $s$ is an integer. Apply the Packing Lemma to pack disjoint disks of radius $3 a_{k}$ whose centers converge to one point $q$ as above.

Let $B_{k}$ be the middle third of these disks so that $\left|x_{k}-q\right|>a_{k}$ for all $x$ in $B_{k}$. Hence $\left|x_{k}-q\right|^{\alpha}>a_{k}^{\alpha}$. But since $\left|\rho_{k}\right| / a_{k}^{s+\alpha} \rightarrow 0$, it follows that $\left|\rho_{k}\right| / a_{k}^{s}<$ $a_{k}^{\alpha}<\left|x_{k}-q\right|^{\alpha}$ for large $k$. Since each $s$ th partial derivative is $C^{\infty}$ smooth away from the limit circle $q \times S^{1}$, we need only check that it satisfies the Hölder inequality for $\alpha$ and for points $\left(x_{k}, t\right)$ in $\beta_{k} \times S^{1}$ and $\left(q, t^{\prime}\right)$ in the limit circle.

However this is the case since

$$
\begin{aligned}
\left|\partial^{s} g_{2}\left(x_{k}, t\right) / \partial x^{s}-\partial^{s} g_{2}\left(q, t^{\prime}\right) / \partial x^{s}\right| & =\left|\rho_{k} / a_{k}^{s} \cdot \partial^{s} \Phi\left(x_{k}^{\prime}\right) / \partial x^{s}\right| \\
& <\lambda \cdot\left|\rho_{k}\right| / a_{k}^{s}<\lambda \cdot\left|x_{k}-q\right|^{\alpha}<\lambda\left[d\left(\left(x_{k}, t\right),\left(q, t^{\prime}\right)\right)\right]^{\alpha} .
\end{aligned}
$$

Hence $g$ is $C^{r}$ smooth. 
Corollary 1. Let $M^{n+1}$ be a $C^{\infty}$ manifold $(n \geqslant 1, n \neq 3)$ and $r<s$ nonnegative real numbers. There exists a $C^{r}$ diffeomorphism of $M^{n+1}$ which is not topologically conjugate to any $C^{s}$ diffeomorphism.

Proof. Choose $\delta$ to lie between $r / n$ and $s / n$, say $\delta=(r+s) / 2 n$, and rotate the $k$ th circle $S_{k}$ in $\mathbf{R}^{n+1}$ through $1 / k^{\delta}$. Apply Theorem 1 to construct an unsmoothable $C^{r}$ diffeomorphism $g$ of $\mathbf{R}^{n+1}$ with compact support. Choose a disk $D^{n+1}$ containing this compact support and a local chart $e\left(\mathrm{R}^{n+1}\right)$ of $M^{n+1}$ where $e$ is a smooth embedding. Let $G$ be the $C^{r}$ diffeomorphism of $M$ induced from $g$ and extended to the complement of $e\left(\mathbf{R}^{n+1}\right)$ by the identity. That is,

$$
G(x)= \begin{cases}\operatorname{ege}^{-1}(x), & x \in e\left(\mathbf{R}^{n+1}\right), \\ x, & \text { elsewhere. }\end{cases}
$$

Suppose $h G h^{-1}$ were $C^{s}$ smooth for some homeomorphism $h$ of $M^{n+1}$. By Lemma 3 we may as well assume that $h$ is close enough to the identity so that $e^{-1} h e \mid: D^{n+1} \rightarrow \mathbf{R}^{n+1}$ is defined and is close to the identity. Then the conjugacy $\left(e^{-1} h e\right) g\left(e^{-1} h^{-1} e\right)$ is not $C^{s}$ smooth by Theorem 1 but it is equal to the $C^{s}$ diffeomorphism $e^{-1}\left(h G h^{-1}\right) e$.

We remark that these examples are not even locally conjugate to a $C^{s}$ diffeomorphism in a neighborhood of a point $x$ in the limit circle. (A $C^{r}$ diffeomorphism $f$ is said to be locally conjugate to a $C^{s}$ diffeomorphism if there exist a homeomorphism $h$ of $M^{n+1}$ and a neighborhood of $U$ of $h x$ in $M^{n+1}$ such that $h f h^{-1}$ restricted to $U$ is $C^{s}$ smooth for some $s>r$.) Suppose $f$ were locally $s$-smoothable in an $n$-ball $U$. Then there is an infinite subsequence $S_{k_{i}}$ of the $S_{k}$ such that for each $i$, more than $1 / 2$, say, of the radial levels of $U$ must have the property that every point in $h S_{k_{i}} \cap U$ at one of these special levels must move less than $\left(1 / k_{i}\right)^{s / n}$ around the core circle under $h f h^{-1}$. One could modify Theorem 1 to show that if such a subsequence did not exist, then $h f h^{-1}$ cannot be $C^{s}$ in $U$ since $\Sigma\left((1 / k)^{s / n}\right)^{n / s}$ $=\infty$. But there are at most $k_{i}^{(r+s) / 2 n}$ points in any orbit of $h S_{k_{i}}$ and since $k_{i}^{(r+s) / 2 n} \cdot\left(1 / k_{i}\right)^{s / n} \rightarrow 0$ the distance between the endpoints of any arc component of $h S_{k_{i}} \cap U$ must tend to zero.

The suspension of a diffeomorphism $f$ of $M$ is the quotient $M \times I /(x, 0) \sim$ $(f x, 1)$. As in [2] one can suspend these diffeomorphisms to obtain unsmoothable foliations or flows.

The proof of the following two corollaries appear in [2] with changes only in notation.

Corollary 2. Let $M^{n+1}$ be a $C^{\infty}$ manifold, $n \geqslant 1, n \neq 3$ and $0<r<s$. There is a $C^{r}$ foliation on $M^{n+1} \times S^{1}$ which is not homeomorphic to any $C^{s}$ foliation.

CoRollaRy 3. If $M^{n+1}$ is compact there is a $C^{r}$ foliation on $M^{n+1} \times S^{1}$ not integrably homotopic to any $C^{s}$ foliation. 
2. Density of unsmoothable diffeomorphisms. Now that the existence of unsmoothable diffeomorphisms has been established, one might ask how common they are. We say that $f$ is $C^{r}$-close to $g$ if the $s$ th derivatives of $f$ are pointwise-close to the sth derivatives of $g$ for $0 \leqslant s \leqslant r$. This defines a $C^{r}$ topology on the space of $C^{r}$ diffeomorphisms of $M$. In this topological space, unsmoothable diffeomorphisms cannot be dense because of the existence of $C^{r}$ structurally stable diffeomorphisms [7]. For there exists a $C^{r}$ neighborhood $U$ of a structurally stable diffeomorphism $f$ such that if $g$ is in $U, g$ is topologically conjugate to $f$. However the following density statement does hold.

COROllaRy 4. Let $M$ be compact. Then the set of $C^{r}$ unsmoothable diffeomorphisms of $M$ forms a dense subset of the space of $C^{r}$ diffeomorphisms of $M$ with the $C^{0}$ topology.

Proof. Let $f$ be a $C^{r}$ diffeomorphism of $M$ and $\varepsilon>0$. Choose $x$ in $M$. Since $M$ is compact there exist integers $p<q$ such that $f^{p}(x)$ and $f^{q}(x)$ are both contained in a small chart of radius $<\varepsilon$. Let $H$ be a $C^{r}$ diffeomorphism of $M$ which sends $f^{q}(x)$ to $f^{p}(x)$ and fixes all $f^{w}(x), p \leqslant w<q$. Then $f_{1}=H \circ f$ is a $C^{r}$ diffeomorphism with periodic point $f^{p}(x)$ of period $q-p$. It is well known that this periodic point can be "blown up" to a periodic disk. That is, $f_{1}$ can be approximated by a $C^{r}$ diffeomorphism $f_{2}$ having a periodic disk with disjoint iterates $A_{i}$. (See Munkres [4, Lemma 1.8], for example.) As in Corollary 1 construct an unsmoothable $C^{r}$ diffeomorphism $g_{1}$ defined in $A_{1}$ which is close to the identity. Define diffeomorphisms $g_{n}: A_{n} \rightarrow A_{n}$ inductively by $g_{n}=f_{2} g_{n-1} f_{2}^{-1}$. Extend the $g_{n}$ to a $C^{r}$ diffeomorphism $g$ of $M$ which is the identity outside $\cup A_{i}$. Finally let $f_{3}=g \circ f_{2}$ and observe that $f_{3}^{q-p} \mid A_{i}=g_{1}^{q-p}$. Hence if $f_{3}$ is smoothable so is $g_{1}^{q-p}$ which is impossible since this merely increases the rotation numbers of $g_{1}$ by a constant factor of $q-p$.

\section{REFERENCES}

1. E. H. Connel, Approximating stable homeomorphism by piecewise linear ones, Ann. of Math. 78 (1963), 326-338.

2. J. Harrison, Unsmoothable diffeomorphism, Ann. of Math. 102 (1975), 83-94.

3. R. C. Kirby, Stable homeomorphisms and the annulus conjecture, Ann. of Math. 89 (1969), 575-582.

4. J. Munkres, Obstructions to the smoothing of piecewise differentiable homeomorphisms, Ann. of Math. 72 (1960), 88.1.

5. J. Palis and C. C. Pugh, Fifty problems in dynamical systems, Warwick Dynamical Systems 1974, Lecture Notes in Math., Springer-Verlag, Berlin and New York, Problem 5.

7. S. Smale, Differentiable dynamical systems, Bull. Amer. Math. Soc. 73 (1967), 747-817.

Department of Mathematics, Princeton University, Princeton, New Jersey 08540

Current address: Department of Mathematics, University of California, Berkeley, California 94720 